\title{
The Machine Design
}

\author{
E. Picasso ${ }^{1}$ and G. Plass ${ }^{2}$
}

${ }^{1}$ Director of the LEP Project, ${ }^{2}$ Leader of the LEP Division

Colliders have become important tools for front-line particle physics research in the last twenty-five years. A collider consists of one or a pair of particle storage rings in which counterrotating beams are brought into collision. Proposals to collide particle beams in this way have been discussed since the mid fifties [1] because only a fraction:

$$
E_{c . m .}=\left(2 m c^{2} E_{\text {lab }}\right)^{1 / 2}
$$

is available in the centre-of-mass of the interaction of two particles of equal rest mass $m$, when a beam of energy $E_{\text {lab }}$ strikes a stationary target, whereas the full energy $2 E_{\text {lab }}$ is available in the collision of the particles of two beams of equal energy.

The design energy of LEP is determined by the masses of the carriers of the weak force. It is set at about $100 \mathrm{GeV}$ per beam, allowing for a certain margin above the energy necessary for the production of pairs of the charged carriers of the weak force, $W^{ \pm}$, which is 165 $\mathrm{GeV}$ in the centre-of-mass, as well as for the search for Higgs particles and toponium, the bound state of top/antitop quarks.

However, much of the physics envisaged will concern the third carrier of the weak force, the neutral intermediate boson $\mathrm{Z}^{\circ}$ with a mass of $93 \mathrm{GeV}$, which is copiously produced at a c.m. energy corresponding to its mass (Fig. 1) [2]. Several thousand $Z^{\circ}$ bosons are expected to be produced per day, allowing the investigation of rare decay modes, the production of hadronic states, and the precision measurement of certain properties of this boson. For the investigation of the $Z^{\circ}$, LEP is first being fitted for acceleration of the nominal beam current to $55 \mathrm{GeV}$. The beam energy will be increased as funding is made available in the years to come.

Several successive versions [3] of a very large $\mathrm{e}^{ \pm}$collider with circumferences varying from 20 to $50 \mathrm{~km}$ were studied at CERN between 1975 and 1980 with respect to their feasibility and cost. The set of parameters according to which LEP is being built and which includes a circumferences of $26.6 \mathrm{~km}$, was chosen such as to reach with normalconducting copper accelerating cavities an energy range where a good rate of $W$ pair production would be obtained. The potential advantage of a superconducting accelerating system was evident, but the technology was not well enough understood at that time (see page 90).

The main parameters of LEP for two energy stages are given in Table 1 and its location is shown in Fig. 2. The LEP Design Report [4] issued in 1984 but still largely valid, contains detailed technical descriptions of all aspects of the project.

The CERN Council approved the LEP project in December 1981 with an anticipated project duration of seven years and a budget, without contingency, of 910 MSF (1981 prices).

\section{SYNCHROTRON RADIATION}

For the accelerator designer, the one basic difference between electron and proton machines of equal energies stems from the different properties of the synchrotron radiation emitted. Synchrotron radiation, which is the current designation for the radiation emitted by charged particle beams travelling on a curved trajectory, entirely determines the design of an accelerator or storage ring for highly relativistic particles. In the case of LEP where the relativistic factor $\gamma=10^{5}$, some of the parameters, and in particular the ring diameter, have been pushed to practical limits, so that it is pretty clear that, for reasons of synchrotron radiation alone, LEP is likely to remain the highest energy circular electron machine ever built.

The average radiation power emitted is given by

$$
\text { where } \begin{aligned}
P & =\left(4 \pi E_{\mathrm{o}} r_{\mathrm{e}} / / 3 e R\right) \gamma^{4} \\
& =E / E_{\mathrm{o}} \\
E & =\text { total energy } \\
E_{\mathrm{o}} & =\text { rest energy } \\
r_{\mathrm{e}} & =\text { classical electron radius } \\
I & =\text { beam current } \\
R & =\text { bending radius }
\end{aligned}
$$

is strongly dependent on the total energy but only linearly on the radius of curvature of the trajectory. This is the only

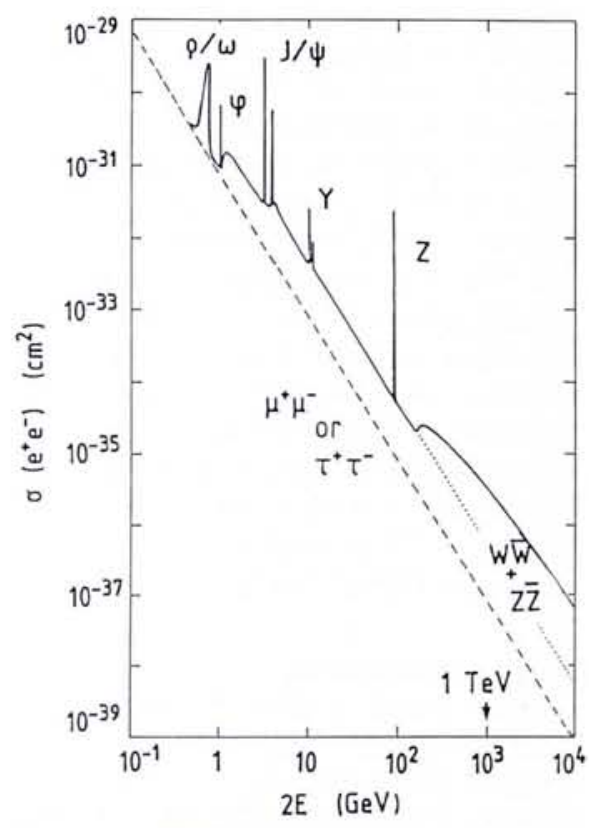

Fig. 1 - Total cross-section of electronpositron interactions.

parameter available to reduce the synchrotron radiation power. In the machine design, a balance must be struck between the cost of a very long tunnel and that of construction and exploitation of the acceleration system necessary to make up for the many megawatts of synchrotron radiation produced.

The critical energy of the radiation, given by

$$
E_{\mathrm{c}}=(3 h c / 4 \pi R) \gamma^{3}
$$

lies, in the case of LEP, in the region of hard X-rays and is therefore an important parameter in the choice of materials and the design of machine components.

Synchrotron radiation also leads to polarization of the beam via the SokolovTernow effect, i.e. an asymmetry in the probability of photon emission according to whether the initial spin state is parallel or antiparallel to the magnetic guide field. The resulting transverse polarization will be useful for the precise determination of the beam energy. With spin-rotating magnets the spins of the beams can be turned into the longitudinal direction for the observation of the interaction of polarized beams. Today, polarization degrees in excess of $50 \%$ appear feasible. 


\section{BEAM PARAMETERS}

\section{Emittance}

All the storage rings that have been built to date are variants of the Alternating Gradient (AG) or Strong Focussing Synchrotron, invented independently by Christofilos and by Courant, Livingston and Snyder [5] around 1950. The focussing forces in the AG synchrotron produce a beam with a characteristic size of a few, up to ten, millimetres in both transverse dimensions; longitudinally, the beam is in general bunched, due to phase focussing by the RF acceleration voltage, the bunch length thus being related to the RF frequency.

The particles of a bunch execute transverse "betatron" and longitudinal "synchrotron" oscillations about their equilibrium positions, described in 6-dimensional phase-space where they occupy a volume called emittance $E$. Beam size in one dimension of real space $\sigma_{x^{\prime}}$ characterized by the r.m.s. value of the amplitude of the particles' oscillations, is given by

$$
\sigma_{x}^{2}=\beta_{x} E_{x}
$$

where $\beta_{x}$ is the amplitude function which varies along the trajectory as a function of the focussing fields.

The designer of a synchrotron chooses sets of parameters and specifies tolerances for the focussing elements such that the oscillations remain bounded, and resonances and instabilities are excluded.

In the highly relativistic regime of LEP, the main driving force of the oscillations, essentially determining the emittance, is the quantized energy loss of the particle, again due to synchrotron radiation.

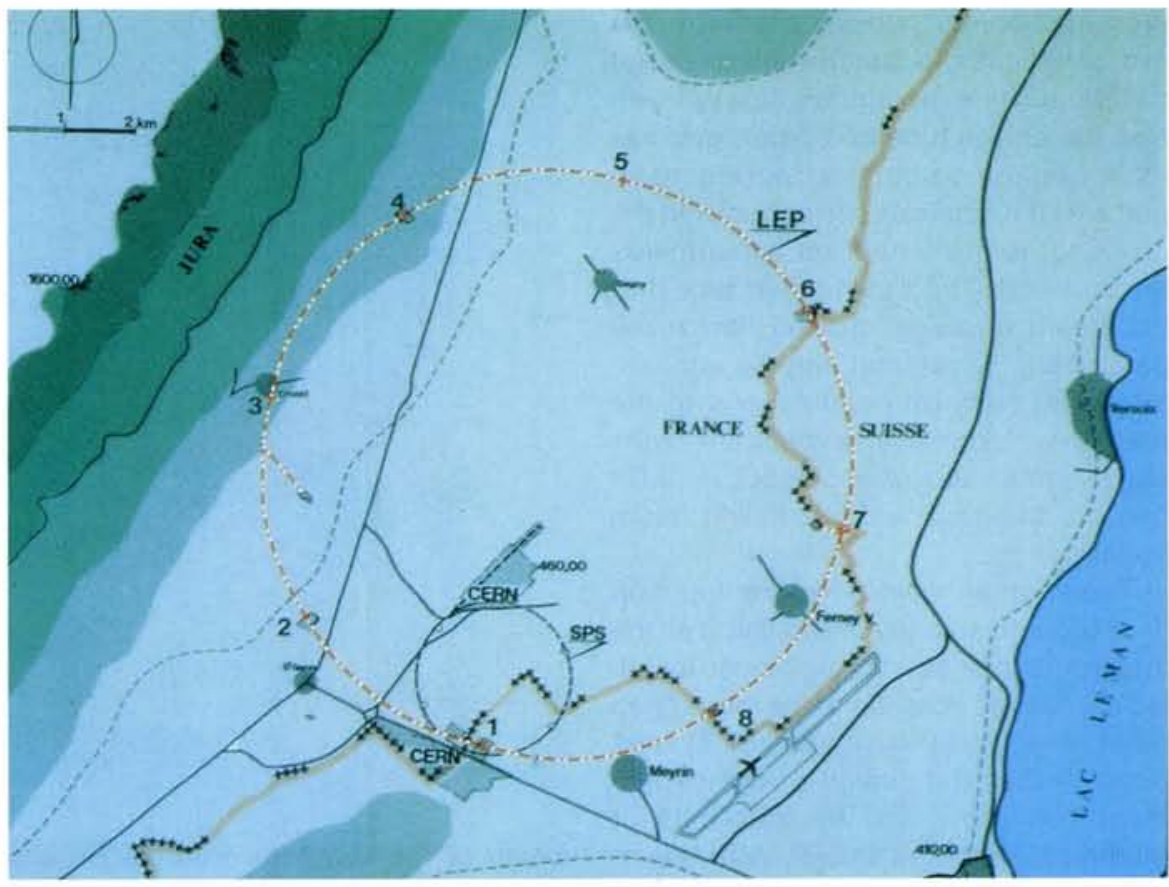

Fig. 2 - The LEP site.

The nominal beam and focussing parameters of LEP are included in Table 1. The regions of stability around the latter are very small in a large machine and will be explored experimentally.

\section{Luminosity}

The performance of a collider is measured in terms of its luminosity

$$
\text { where } \begin{aligned}
L= & N^{2} f / 4 \pi k \sigma_{x} \sigma_{y} \\
f= & \text { particles per beam } \\
= & \text { revolution frequency } \\
& \text { in the ring } \\
k= & \text { number of bunches } \\
\sigma_{x} \sigma_{y}= & \text { r.m.s. beam radii in } \\
& \text { the transverse planes }
\end{aligned}
$$

\section{Table 1 - LEP Parameters}

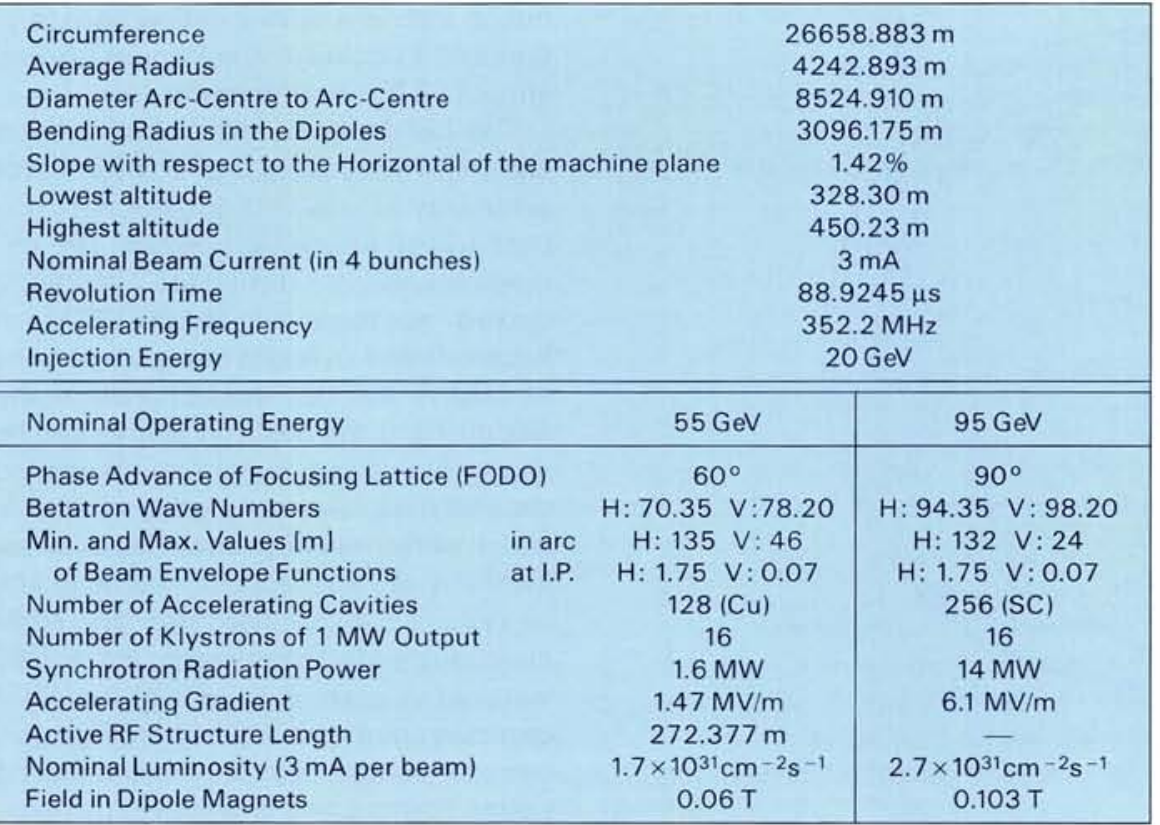

which, multiplied by the interaction cross-section, gives the interaction rate.

It is of interest to express the luminosity as a function of the "beam-beam tuneshift", a parameter which describes the mutual focussing effect of the colliding bunches in terms of the change of the number $Q$ of transverse betatron oscillations of the particles around the circumference. In the vertical plane, and for flat beams $\left(\sigma_{y} \ll<\sigma_{x}\right)$, this is where $\beta_{y}$ is the vertical amplitude function at the interaction point

hence $\begin{aligned} \Delta Q_{y} & =N r_{e} \beta_{y} / 2 \pi k \gamma \sigma_{x} \sigma_{y} \\ L & =N \gamma \Delta Q_{y} / 2 r_{e} \beta_{y}\end{aligned}$

or, introducing beam current $l=N e f$

$$
L \quad=\mid \gamma \Delta Q_{y} / 2 e r_{e} \beta_{y}
$$

According to equ. 8 , three parameters determine, for a given energy, the attainable luminosity: $I, \Delta Q_{y}$, and $\beta_{v}$.

The beam current $I$ is limited by the electrodynamic interaction between the particles in a bunch, between successive bunches in the beam, and by the interaction of the beam with its environment, i.e. the vacuum chamber and the residual gas in it. The first mentioned interactions can provoke beam instabilities, leading to blow-up and partial or catastrophic losses of the beam. The collisions with the gas molecules remaining, even at the low pressures achieved in storage rings, determine the rate of current decay. The beam lifetime in LEP is expected to be of the order of several hours.

The beam-beam tuneshift $\Delta Q_{y}$ depends on the number of beam crossings around the circumference and inversely on the energy. Electrostatic deflectors are therefore installed near each of the 
crossing points which separate the orbits vertically so that the beams do not collide in the experiments during injection (when the tuneshift parameter has its maximum value) and acceleration, and are kept separate permanently in the crossing points where no experiments are installed. The beam-beam tuneshift per beam crossing depends on many parameters which can only be accounted for in computer simulations of the machine. Extensive simulation runs show that a value of about $\Delta Q_{y}=0.04$ can be admitted without losing beam stability.

The vertical beam envelope function $\beta_{y}$ is brought to a minimum value at the interaction point by strong lenses installed as close as possible to the interaction point. The limit is given by the closest position that the design of the experiment can allow and by the limits of magnet technology. In LEP, with superconducting lenses installed at $5 \mathrm{~m}$ from the crossing point, a $\beta_{y}$ value of $0.07 \mathrm{~m}$ can be achieved, yielding ribbon-shaped beams of $12 \mu \mathrm{m}$ height and $300 \mu \mathrm{m}$ width, while the length of the bunches is $18 \mathrm{~mm}$. Owing to the discontinuous replacement (by the acceleration systems in Points 2 and 6 ) of the energy lost by synchrotron radiation, the orbits of the electrons and the positrons are not rigorously identical and would miss vertically. They are steered into collision by the fine adjustment of the above-mentioned electrostatic deflectors. With the above nominal values, a luminosity of $1.7 \times 10^{31} \mathrm{~cm}^{-2} \mathrm{~s}^{-1}$ is expected at $55 \mathrm{GeV}$.

Reducing background, i.e. the number of particles that might originate from the

Fig. 4 - Tunnel cross-section.

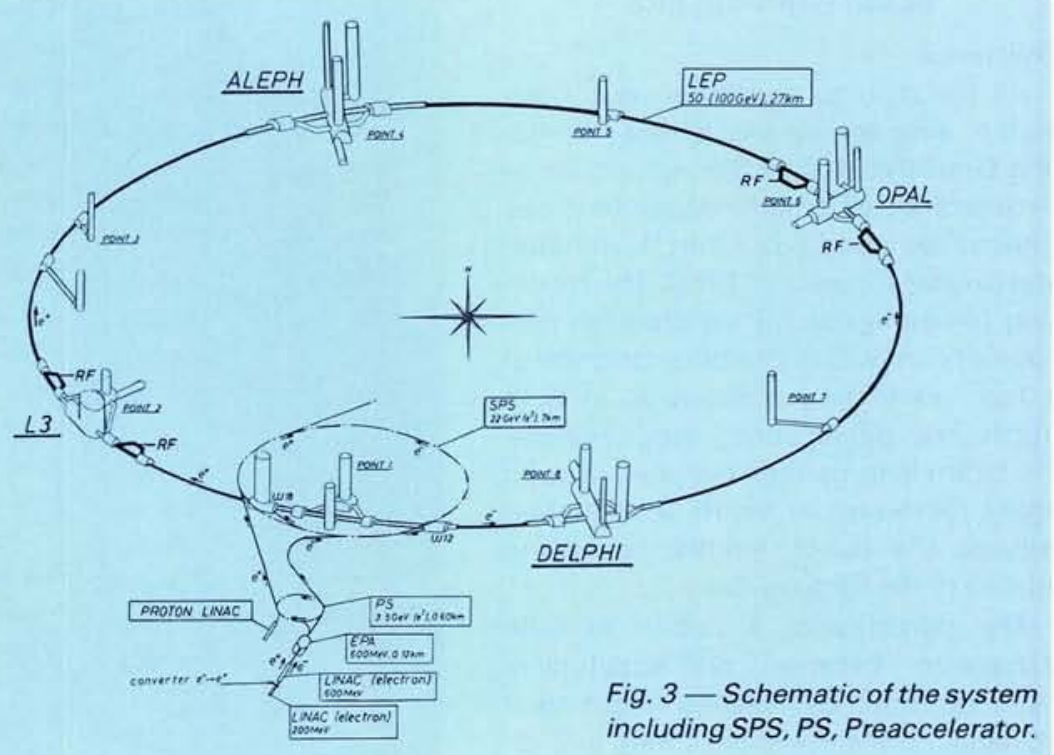

beam or the synchrotron radiation and simulate, in the detector, tracks coming from collision events, is another important design consideration.

A first important reduction of the photons due to synchrotron radiation is obtained by the introduction of a very soft bend at the end of each machine octant: a series of magnets with a field only 10 percent of the standard arc dipoles will reduce both the number and the energy of the photons which could reach the experiment. Together with this goes the installation of a system of collimators which will further absorb photons and also electrons which can travel along the vacuum chamber or be created by Compton scattering with the residual gas.

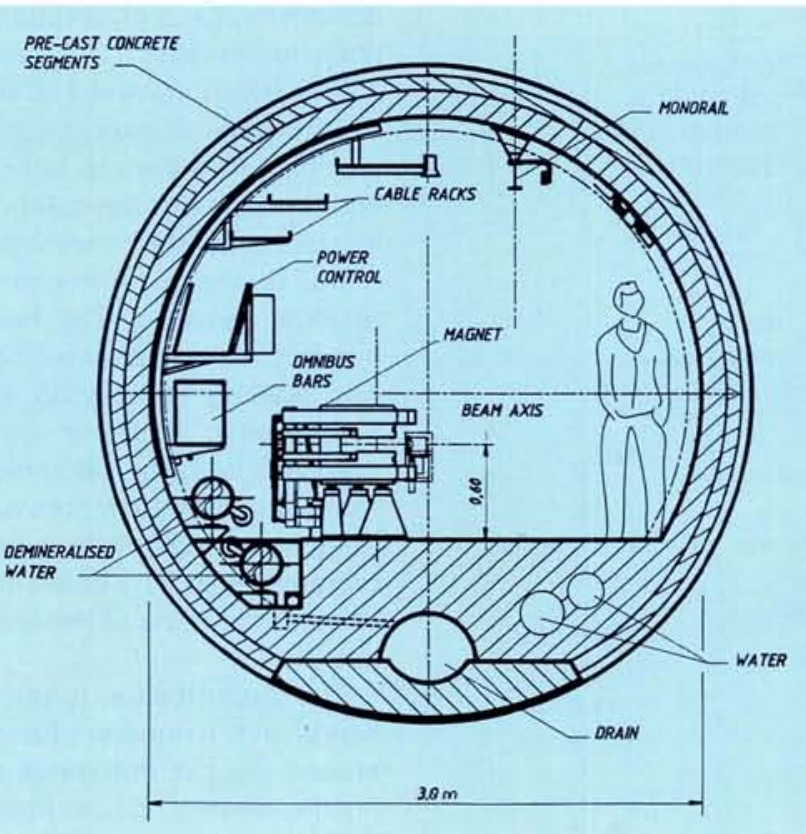

\section{THE LEP COLLIDER}

\section{Overview}

LEP has been sited at an average depth of about $100 \mathrm{~m}$ under the area adjacent to the CERN main laboratory sites (Fig. 2). As can be seen, the tunnel passes under normally inhabited land, belonging to France over three quarters of the circumference, and to Switzerland for the remaining quarter. The tunnel and the many associated caverns have been drilled through the bedrock of this area, consisting of molasse for most of the circumference and of limestone for about $3 \mathrm{~km}$ near the Jura mountains. Tunnel and caverns are linked to the surface by a total of 18 shafts. At the surface, patches of land totalling about 25 ha were acquired around the access points. Both the overall machine location and the local building layout at each surface area were carefully adjusted so as not to interfere with existing construction and to achieve a minimum general impact on the environment.

The LEP magnet lattice has a basic eight-fold symmetry, broken by a mirror symmetry of adjacent octants with respect to the interaction points. The circumference is composed of eight curved sections of about $2800 \mathrm{~m}$ length, linked by eight straight sections of $500 \mathrm{~m}$ length. The bunches of the electron and the positron beams can be allowed to collide at eight points around the machine, and many other characteristics of the machine obey this or lower symmetries. The access shafts to the tunnel are concentrated near the eight crossing points. Experiments are initially installed in caverns at four of the eight crossing points, while accelerating cavities are located near only two crossing points, Points 2 and 6 , where extra paral- 


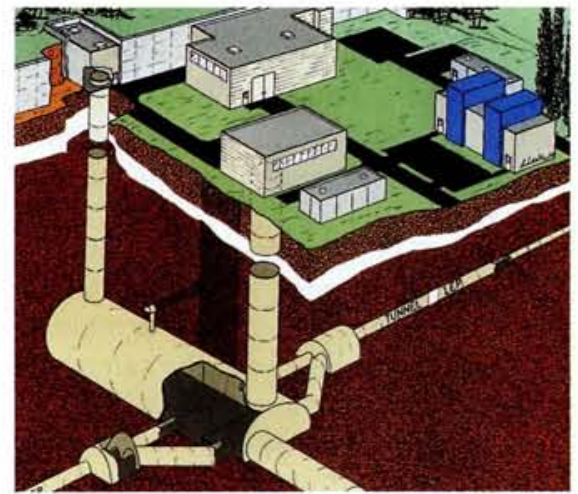

Fig. 5 -Artist's view of crossing point.

Fig. 6 (Right) - Aerial view of one of the surface areas.

lel galleries have been dug to house the $\mathrm{RF}$ transmitters.

Fig. 3 gives a schematic overview of the project including the pre-accelerators and transfer lines for the beams. Fig. 4 shows the cross-section of the machine tunnel. Fig. 5 shows schematically the layout of tunnels and caverns near one of the crossing points where both an experiment and the acceleration system will be located. An aerial view of one of the surface areas is shown in Fig. 6 .

The design and construction of a large new system like LEP requires the development of many subsystems and techniques required to achieve the desired goal. Many of the components or subsystems are necessary a hundred fold or in thousands around the circumference and the unit prices of these items tend to become very important for the project budget. Years of design effort were invested in order to reduce their cost.

It is not possible to present here in detail all the systems that together will make up the LEP collider. We will therefore only discuss a selection of components or systems which are characteristic of the LEP collider.

\section{Magnets}

LEP consists, for about $20 \mathrm{~km}$ of its circumference, essentially of dipole magnets (1672 units of $12 \mathrm{~m}$ in length) which keep the beam on its circular path. An unusually low bending field (about $0.1 \mathrm{~T}$ ) is required even at top energy, the bending radius having been chosen to be very large in order to limit the synchrotron radiation. Classical dipole cores would have contained an unnecessary mass of poorly used magnetic steel, so instead a novel design with an optimum steel filling factor of only 0.27 was adopted [2]. The magnet cores are composed of a stack of lamina-

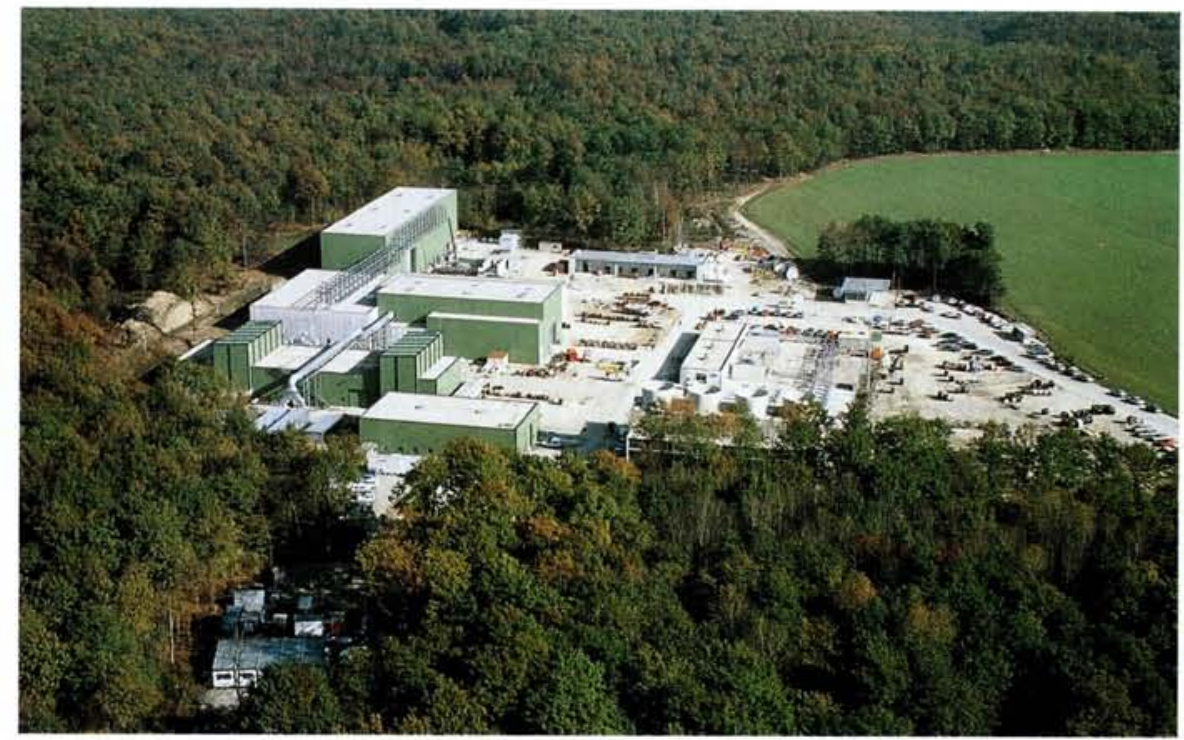

tions, $1.5 \mathrm{~mm}$ thick and separated by $4 \mathrm{~mm}$ gaps filled with cement mortar. Four pre-stressing rods act on two endplates and compress the core so that it behaves like a pre-stressed concrete beam (Fig. 7). Compared to classical cores, a saving of about $40 \%$ in cost and weight has been achieved.

Many other types of magnets must be produced to very tight tolerances and installed in the LEP tunnel: 816 quadrupoles, 504 sextupoles, about 500 orbit correction dipoles, and others. Special mention must be made of the eight superconducting quadrupole magnets necessary to focus the beam at the interaction point. Their position inside the detector set-up requires a special design of the current feeder and the cold/warm transition and long flexible transfer lines of original design [6] for the liquid $\mathrm{He}$.

\section{Accelerating System}

LEP being both an accelerator and a storage ring, an acceleration system operating at a frequency of $352 \mathrm{MHz}$ is required to bring the beam from injection $(20 \mathrm{GeV})$ to the operating energy and to make up for the energy lost to synchrotron radiation. With copper cavities as installed in Phase 1, a large fraction of the $16 \mathrm{MW}$ input energy is lost in the walls. In order to limit these losses, each of the five-cell accelerating cavities of active length $2.125 \mathrm{~m}$, is coupled [3] to a low-loss spherical resonator (Fig. 8) with a slightly different resonant frequency. The system is excited so that the field energy will oscillate between the two cavities with a frequency given by the beating between the two frequencies. This scheme reduces the ohmic losses in the cavity walls sub-

Fig. 7 -Dipole core structure.

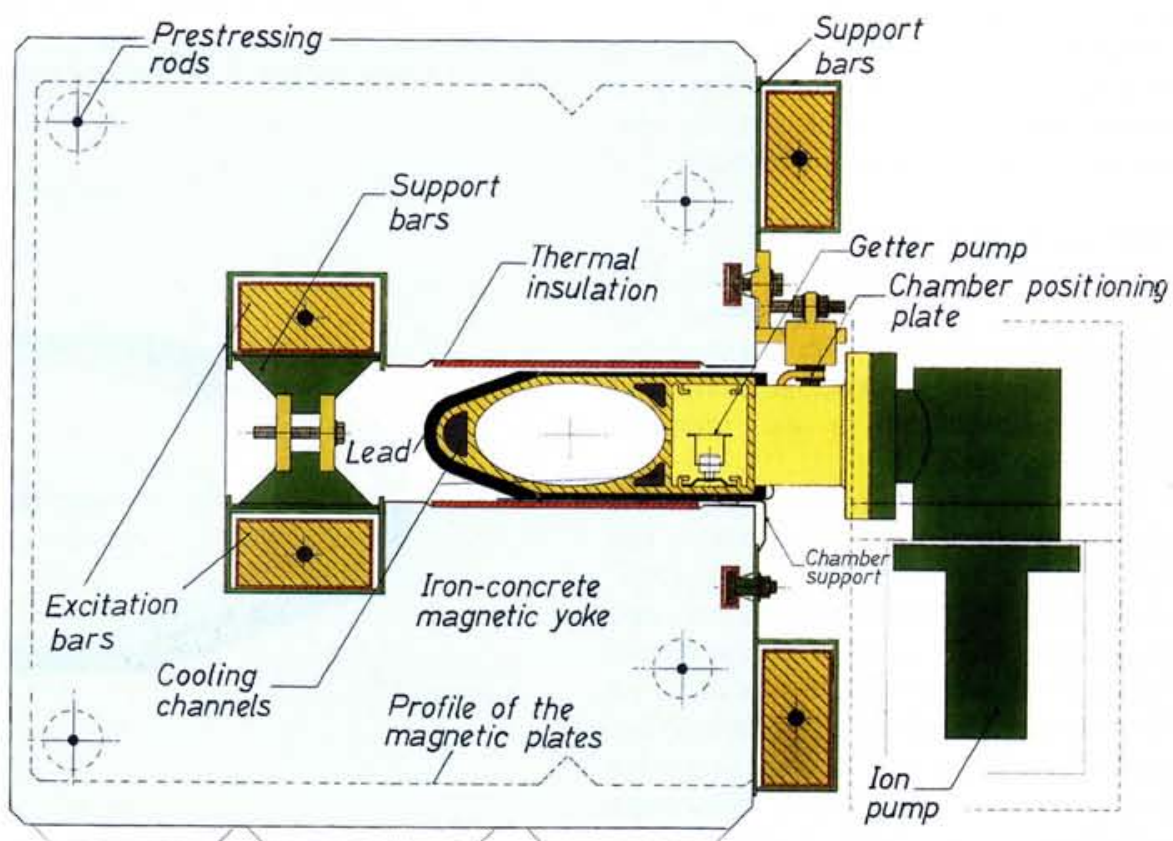




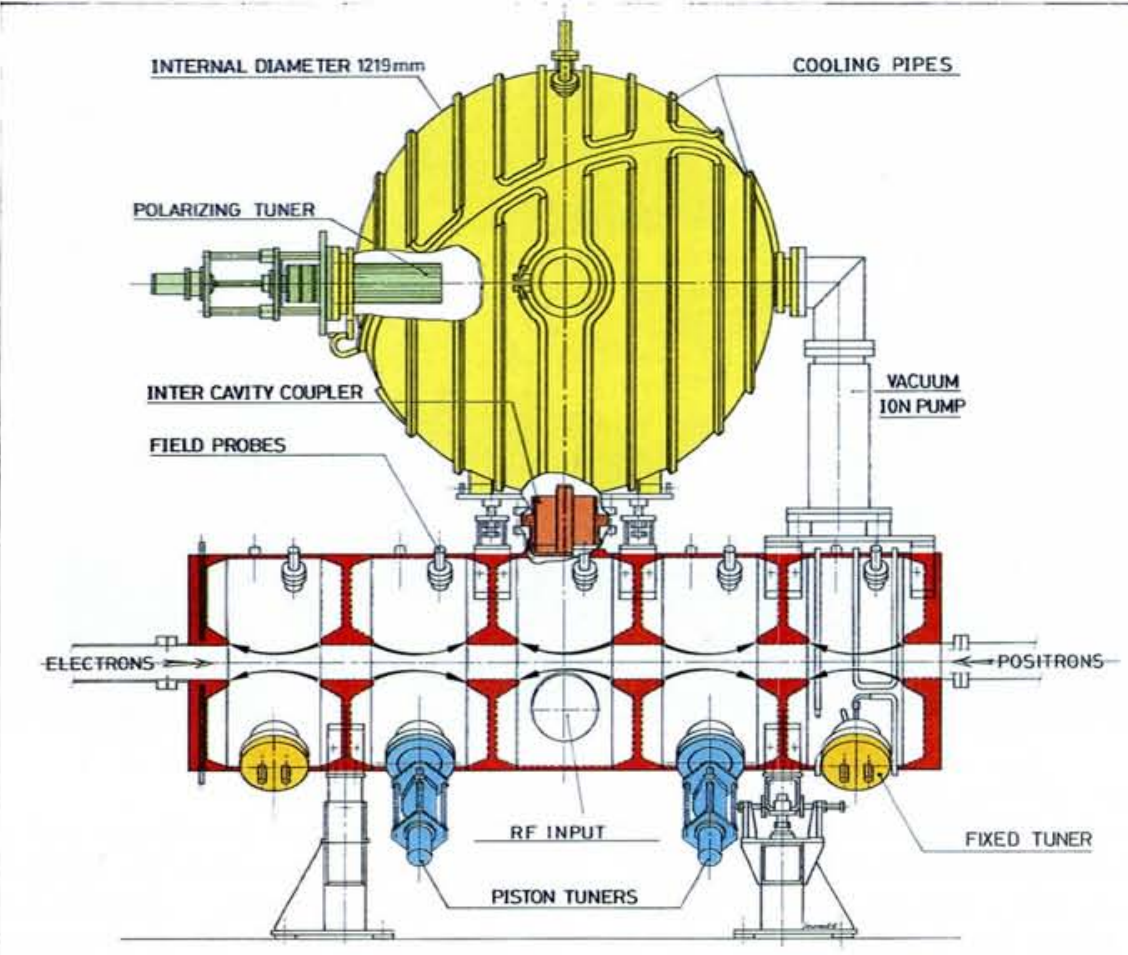

Fig. 8-Accelerating and storage cavity system.

stantially and the total power required by a factor 1.6.

In order to reduce ohmic losses to a very low figure for the planned increase of the beam energy, the use of superconducting cavities is foreseen (q.v.).

The total number of 128 cavities is divided into groups of 16 , each of which is powered by a pair of specially developed $1 \mathrm{MW}$ klystrons whose efficiency has been pushed to $68 \%$ for this purpose. The cavities are installed on both sides of the experimental area in Points 2 and 6 , which are about $8.5 \mathrm{~km}$ apart. The klystrons are driven by a stabilized master oscillator, phase synchronization to about $1^{\circ}$ tolerance over the distance being achieved by a phase-compensated link of monomode optical fibres [7].

\section{Vacuum System [8]}

Synchrotron radiation and the low bending field have strongly influenced the design of the LEP vacuum chamber which is made of an extruded aluminium profile covered with a lead radiation shield (Fig. 9).

The strong desorption, particularly during early operation, of gas from the vacuum chamber walls hit by synchrotron radiation, requires a distributed pumping system in view of the length $(12 \mathrm{~m})$ and the limited conductance of the standard vacuum chambers. Whereas in other electron storage rings this is achieved by linear sputter-ion pumps operating in the field of the bending

Fig. 9 -Cross-section of the vacuum chamber. migrate into the bulk of the material, liberating the surface for a new cycle of pumping. It is estimated that during 20 years of LEP operation, between 10 and 20 reconditioning operations should be necessary.

As the NEG pump does not pump rare gases or methane, small sputter-ion pumps $(30 \mathrm{l} / \mathrm{s})$ are mounted in addition every $20 \mathrm{~m}$. They serve at the same time for pressure monitoring. The LEP vacuum system does not include permanent primary pumps. After installation, or after an intervention on the system, the chambers are pumped down by mobile pumping stations while being baked-out at $150^{\circ} \mathrm{C}$. They are then sealed off. According to results obtained so far, the LEP pumping system should be able to produce an average static pressure of $10^{-9} \mathrm{~Pa}$ one day after bakeout and a pressure in the low $10^{-10} \mathrm{~Pa}$ range after a few weeks.

\section{Beam Instrumentation}

LEP will be equipped with the complete set of instruments essential for understanding and the successful operation of a new particle accelerator [9]. Around the circumference, 504 beam position monitors, calibrated to $0.1 \mathrm{~mm}$ precision and connected to the Token Ring data highway (q.v.) will be installed. Precise beam current measuring transformers have been developed as well as a UV telescope and wire-scanners for the determination of the beam size, the latter allowing in particular the investigation of the "tails" of the beams which can be very important for understanding the background and beam lifetime problems. Solid-state X-ray monitors will be used for beam-height measurements and X-ray correlators for the measurement of the bunch length.

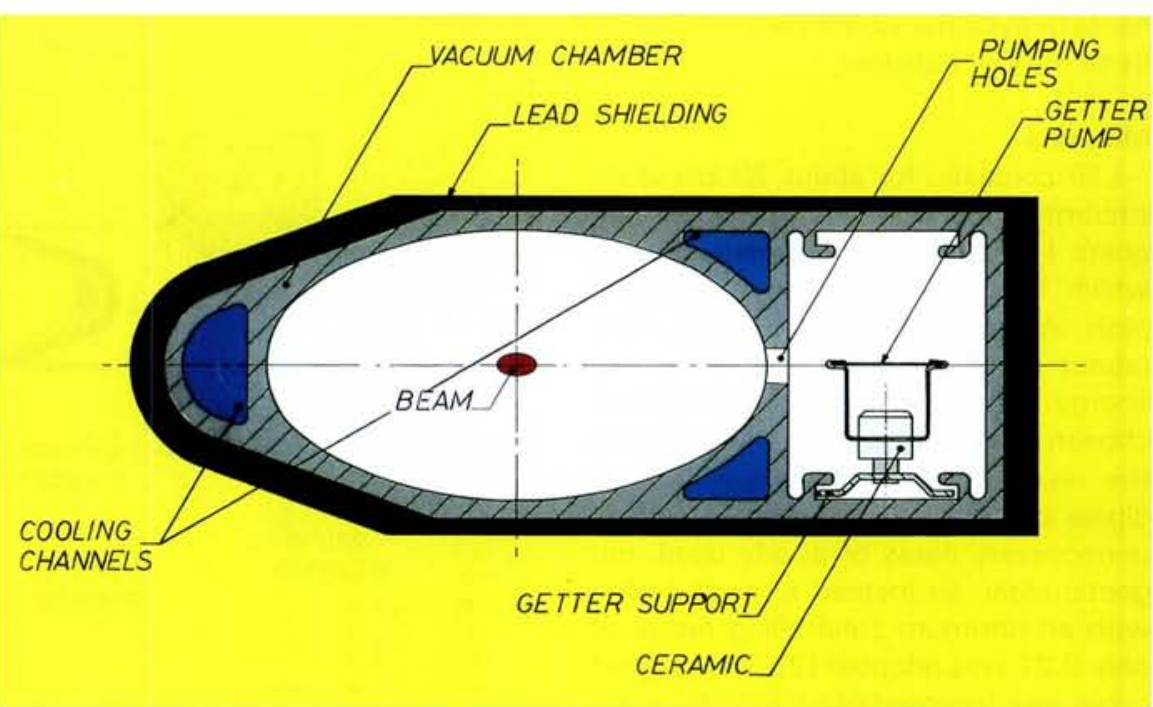


The interaction rate will be measured with small calorimeters mounted on collimators inside the vacuum chamber and measuring electrons from Bhabha scattering at the interaction region.

There will be 40 collimators installed to protect the experiments and the electrostatic deflectors against synchrotron radiation, and a set of 16 collimators are provided as protection against Compton-scattered electrons from the arcs. A further set of eight collimators will be used to define the machine aperture.

\section{Power Converters}

A large number of power converters $[10]$ is required in order to supply the systems described in the previous sections with power from the mains. In total, 750 units are needed for the magnet and RF systems, plus 550 small units for the ion pumps. Their specifications cover a very wide range: the output power ranges from a few hundred watts to several megawatts, the highest output voltage is $100 \mathrm{kV}$ (for the RF klystrons), and the highest current required is $33 \mathrm{kA}$ (for one of the experiments). The best specified current stability is $\pm 5 \times 10^{-5}$ (for the main dipole and quadrupole strings) and current regulation range of 1:1000 is specified for a smooth passage through zero of the bipolar supplies of the 544 corrector magnets.

Switch-mode converters are used for ratings up to $37 \mathrm{~kW}$ because of their compactness and good efficiency and thyristor-controlled circuits for higher output power. The microprocessor-controlled electronics have been standardized throughout the whole range so as to achieve low production and maintenance cost.

The reliability of this equipment as that of many others will obviously be crucial for the success of LEP.

\section{Controls}

Many of the design choices for the LEP control system [11] have been dictated by the size and topology of the project. Thus, the cost of laying many cables around the $27 \mathrm{~km}$ circumference led to the decision to replace cables by a Time-Division-Multiplex (TDM) system as used in telephone trunk lines to transport on one cable as many as possible of the necessary signals and communications. A small number of cables was authorized only for those purposes where "hard-wired" links are unavoidable, i.e. for safety links. The TDM system consists of two coaxial cables going around the ring (glass-fibres could deteriorate in the radiation environment of the ma- chine), each transmitting data at 32 $\mathrm{MB} / \mathrm{s}$ rate.

The geographical size of the project also suggests a distributed computer system, the computers being installed near the equipment, i.e. in technical areas distributed along the circumference, on the one hand, and near the control centre which has been integrated into the control room of the SPS, on the other hand.

The IBM Token Ring system has been chosen for linking all the computers into a network. The Token Ring in principle consists of two ring cables into which computers can be joined at any place. For the LEP case, the Token Ring communication travels via the TDM system, where two $8 \mathrm{MB} / \mathrm{s}$ channels are reserved for this purpose.

About 150 computers are deployed in the network, of which 16 are Apollo workstations, used as man-machine interfaces.

The machine components or subsystems are linked to the local computers ("process control assemblies" - PCA) via multidrop buses (MIL/STD-1553 B), which support up to 30 connections each.

\section{7 kilometers of LEP vacuum chamber maintained at $10^{-12}$ mbar}

This considerable result has been achieved mainly thanks to the pumping action of a non-evaporable getter (NEG) strip which runs along most of the $27 \mathrm{~km}$ of the LEP vacuum chamber. The strip was developed by SAES GETTERS S.p.A., the world leading company in the field of gettering, other vacuum related technologies and pure gas handling.

SAES GETTERS and CERN have worked together to optimize the pumping performance of the getter strip, whose characteristics are: high

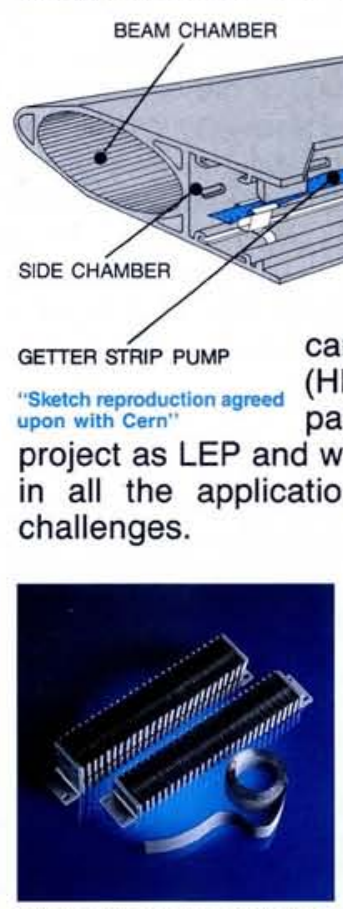

Wide getter strip and Wafer modules

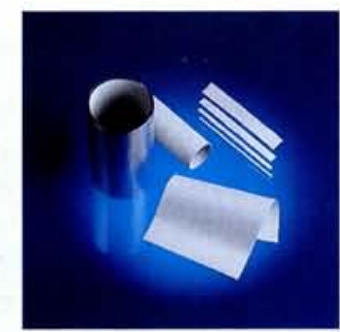

High Porosity Thick Film getters distributed pumping speed, constant pumping over a wide pressure range $\left(10^{-5}-10^{-12}\right.$ mbar), cleanliness and no power requirements for operation.

These peculiar features are present also in other SAES GETTERS products such as Wafer modules, SORB-AC cartridge pumps and High Porosity Thick Film (HPTF) getters. SAES GETTERS is proud to have participated, as a supplier, in such an important FULFILLING TOMORROW NEEDS ... TODAY

\section{Saes SAES GETTERS getters \\ EULEILLING TOMORROW NEEDS ... TODAY

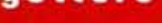 \\ Headquarter: \\ 20151 Milano - ITALY \\ Via Gallarate, 215 \\ Tel. (2) 30201 (20 lines) \\ Telex 331108 - Telefax (2) 33403636}

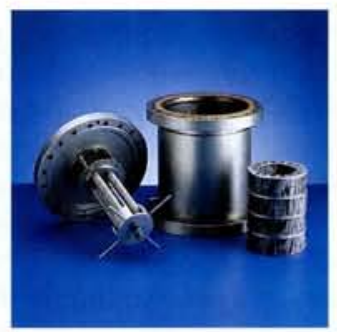

All metal SORB-AC ${ }^{\circ}$ pump

Companies in United States, Japan, Korea, West Germany, France, United Kingdom 
The LEP controls network must communicate with the pre-accelerators, notably the SPS, and the CERN computer centre as shown in Fig. 10.

\section{Injection System}

It was decided during the project definition phase, in order to make full use of the infrastructure available in the laboratory, to use the two proton synchrotrons, the SPS $(450 \mathrm{GeV}$ protons, first operated in 1976) and the PS (28 GeV protons, in continual operation since 1959), as injectors for LEP. Their top energies for electrons were set at 20 $\mathrm{GeV}$ (which is then the injection energy for LEP) and $3.5 \mathrm{GeV}$ respectively, in order to limit the synchrotron radiation power in these machines.

The PS receives electrons and positrons from a set of three new machines which were built in collaboration with the Laboratoire de l'Accélérateur Linéaire at Orsay (France):

* a $200 \mathrm{MeV}$ electron linac which delivers currents of up to $2 \mathrm{~A}$ onto a converter target for positron production (in fact, $\mathrm{e}^{ \pm}$pair production by shower photons)

* a $600 \mathrm{MeV}$ linac for the acceleration of electrons or positrons

* a $600 \mathrm{MeV}$ storage ring that acts as a buffer between the $100 \mathrm{~Hz}$ cycling rate of the linacs and the approximately $1 \mathrm{~Hz}$ cycling of the PS.

Fig. 11 shows a general view of the linac accelerating system. Space does not permit us to go more into the detail of this system, which has been documented elsewhere [12].

Advanced injection and ejection equipment was added to the PS and the SPS for the transfer of the particles between the machines as well as new acceleration and beam observation systems dedicated to electrons and positrons.

Beam transport channels about 400 and $650 \mathrm{~m}$ in length respectively guide the positrons and the electrons from the SPS down to LEP which is located about $40 \mathrm{~m}$ lower. Signals derived from the synchronization of the LEP acceleration system are used to trigger the transfer of bunches from the SPS so that these are caught in the correct RF buckets of the counter-rotating LEP beams and collide in the centres of the four detectors.

The injector complex can provide the $\mathrm{e}^{ \pm}$beams in parallel to accelerating protons for fixed target physics: Fig. 12 shows the system of interleaved cycling which allows LEP and fixed target physics to run simultaneously with a very small loss in average flux for the latter.

In order to fill LEP, bunches of electrons and positrons are accelerated in

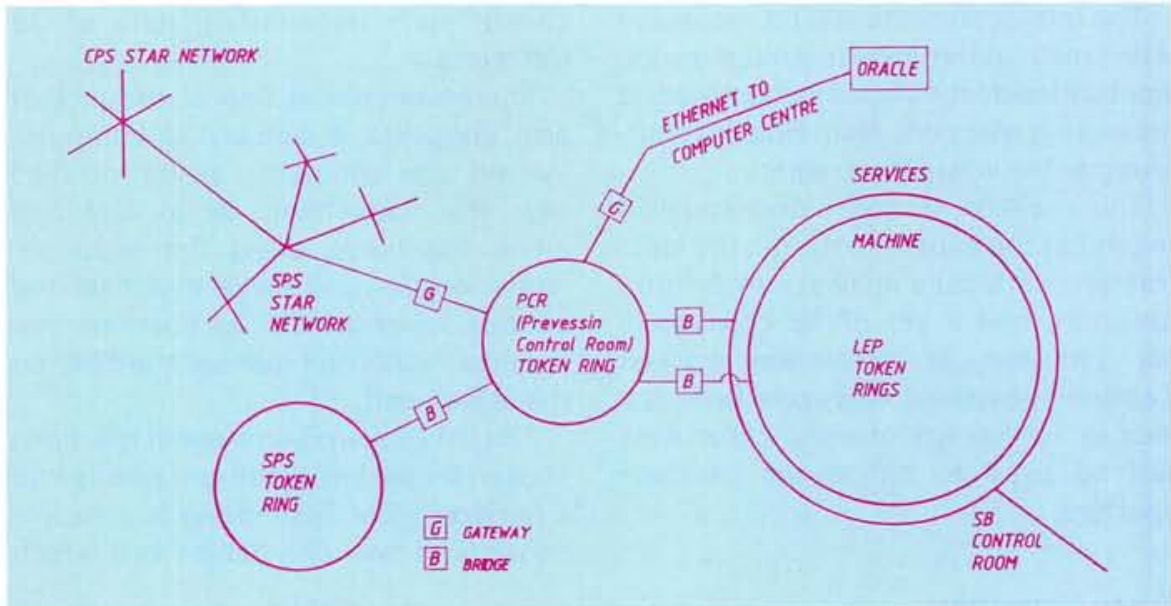

Fig. 10-SPS/LEP control networks.

the SPS in four $20 \mathrm{GeV}$ cycles located between proton cycles, transferred to LEP, and stacked in four bunches in each beam. About 50 SPS cycles, i.e. about 12 minutes, will be necessary to obtain the nominal LEP current of $3 \mathrm{~mA}$.

\section{INFRASTRUCTURE}

More than half of the LEP Project budget has been spent for items under this heading. Whereas the need for underground tunnelling for housing a machine the size of LEP is rather apparent, the size of the necessary infrastructure networks for providing power and cooling as well as appropriate conditions for people and equipment may be rather less evident. Again, we can only scan through some of the gross features of the LEP infrastructure within the bounds of this report.

\section{Site}

CERN has a long standing experience with the stability of the local bedrock and with the execution of civil engineering operations in it. Very extensive stu- dies of its stability have been made, ever since the decision to locate CERN near Geneva was taken in 1953. These and the unperturbed operation of the PS and the SPS gave the confidence that even a machine of the size of LEP could be located in the Geneva area and the Pays de Gex (France) without major stability risks.

The local bedrock, molasse, is a layered material consisting of sandstones of varying properties and clays. Though inhomogeneous on a small scale, the molasse of the Lake Leman valley is sufficiently continuous on the scale of kilometres to provide a very good support for our accelerators. As the molasse has no cracks and contains only traces of water or hydrocarbon, the tunnelling and other excavation operations are fairly predictable.

For LEP it was envisaged for the first time to extend the accelerator tunnel under the Jura mountain because of the size, in particular, of the early versions of the project. Jurassic limestone is known to carry water in caverns or in a network

Fig. 11 - An overall view of the $600 \mathrm{MeV}$ Linac.

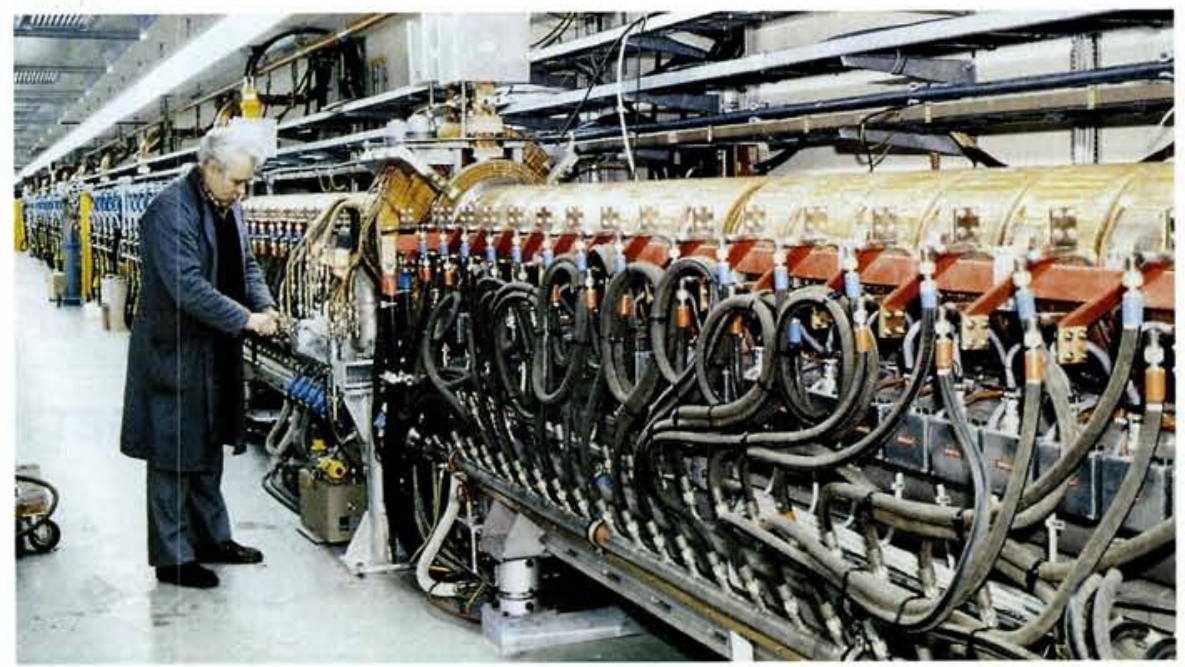




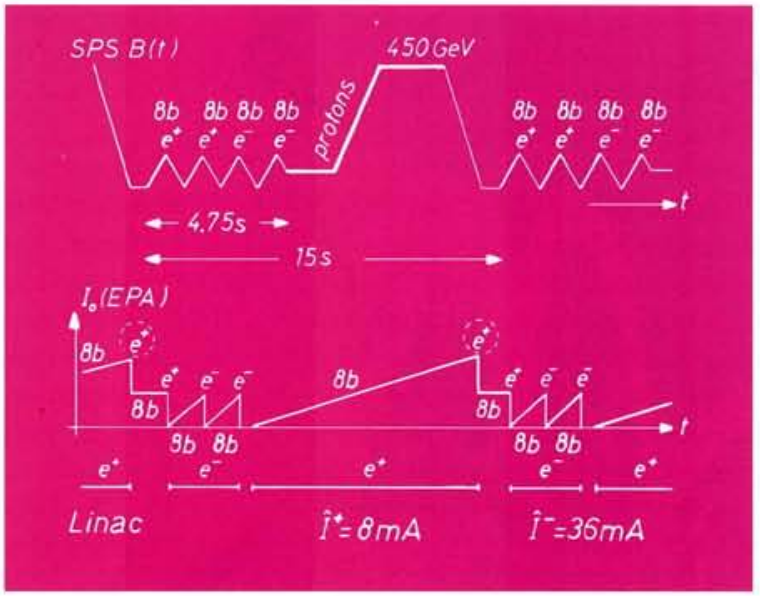

Fig. 12 - Schematic of the SPS cycles.

of cracks which can communicate over hundreds of meters. Flow rates up to several cubic metres per second have been measured during accidental leakages in traffic tunnels in the Jura.

Particularly delicate and unusual is a tunnel like the LEP tunnel without a natural gravitational outlet and which must follow a precisely prescribed trajectory. This implies the risk of a complete flooding of the tunnel and an impossibility of deviating the tunnel, as is often done when geological obstacles are met. Therefore, a final effort was made in the months prior to project authorization, once the size and the geometry of the project had been determined, to reduce the tunnel length under the Jura. The final decision was for the position indicated in Fig. 2, and for the installation at the point of attack of the Jura section, of a set of powerful pumps with $2000 \mathrm{~m}^{3} / \mathrm{h}$ capacity at $75 \mathrm{~m}$ head in order to cope with eventual water problems. This position also preserves the possibility of creating electron-proton collisions between LEP electrons and $300 \mathrm{GeV}$ protons in a by-pass of the SPS, in LEP Point 1.

After extensive campaigns of test borings it was decided to incline the plane of LEP by $1.4 \%$ with respect to the horizontal, so that all caverns are located in solid rock with adequate coverage below the moraine, while the depth of the shafts is limited to about $150 \mathrm{~m}$.

\section{Civil Engineering}

Underground civil engineering [13] for the LEP Project called on a large variety of techniques.

Starting out from the surface, 18 shafts of between $5 \mathrm{~m}$ and $23 \mathrm{~m}$ in diameter and between 50 and $150 \mathrm{~m}$ in depth had to be sunk in order to reach the level of the machine tunnel. Excavation was straightforward where the shafts traversed the molasse, whereas special techniques were necessary in each case, would have been extremely difficult had the tunnel been blocked by the 150 metre length of a boring machine and its associated equipment.

A variety of roadheader-type machines were used for the excavation of the caverns of various sizes, which could almost all be located in the molasse. The caverns are basically self-supporting after allowing for a certain convergence of the rock after excavation (Fig. 15). However, as the molasse degrades after exposure to moisture, all tunnels and caverns are lined with mass concrete. In the limestone, the concrete shell of the tunnel is dimensioned so as to withstand more than 15 bars of water pressure, which corresponds to the height of the overlaying rock.

The LEP Project also includes the construction of some 70 surface halls of various sizes near the access points (Fig. $5)$, which serve for the transfer of material down the shafts and house the various services necessary for the collider and the experiments.

\section{Survey [14]}

The task of guiding the tunnelling machines on their path to within one centimetre or so, and to align the collider inside its tunnel to millimetre precision on a scale of several kilometres, is certainly one of the more fascinating aspects of this project.

The basic reference for this work is provided by a network between the hills surrounding the site (Fig. 16) with baselines of up to $13 \mathrm{~km}$ length. The latter were measured with $10^{-7}$ precision with the help of a two-wavelength laser interferometers (Terrameter) which permits cancellation of the variation of the optiaction, which took about six months in

Fig. 13 - A tunnelling machine breaking through at the end of an octant.

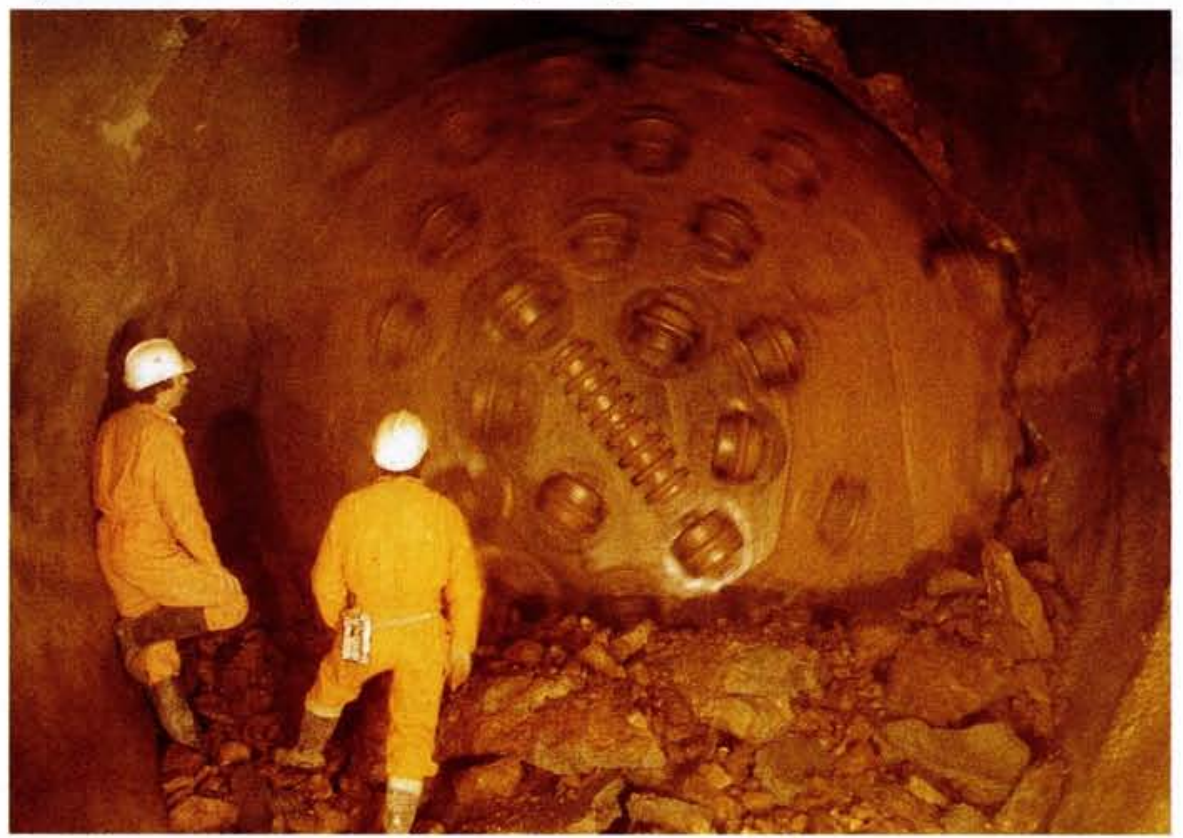


cal properties of the air. In recent years it has also been possible to check these measurements with satellite observations, using the NAVSAT satellite system, which gave excellent agreement with the earlier method.

Reference points are then created near each shaft, and the measurement must be transferred down to monuments at the shaft bottom. The tunnelling machines are guided by laser beams which are realigned daily with the help of a gyro-theodolite, an instrument that is carefully aligned to the North on surface before it is brought down the shaft.

The positions of the machine elements in the tunnel are determined by a chain of quadrilateration figures between the monuments at two adjacent

Fig. 14 (Right) - Water problems in the Jura octant.

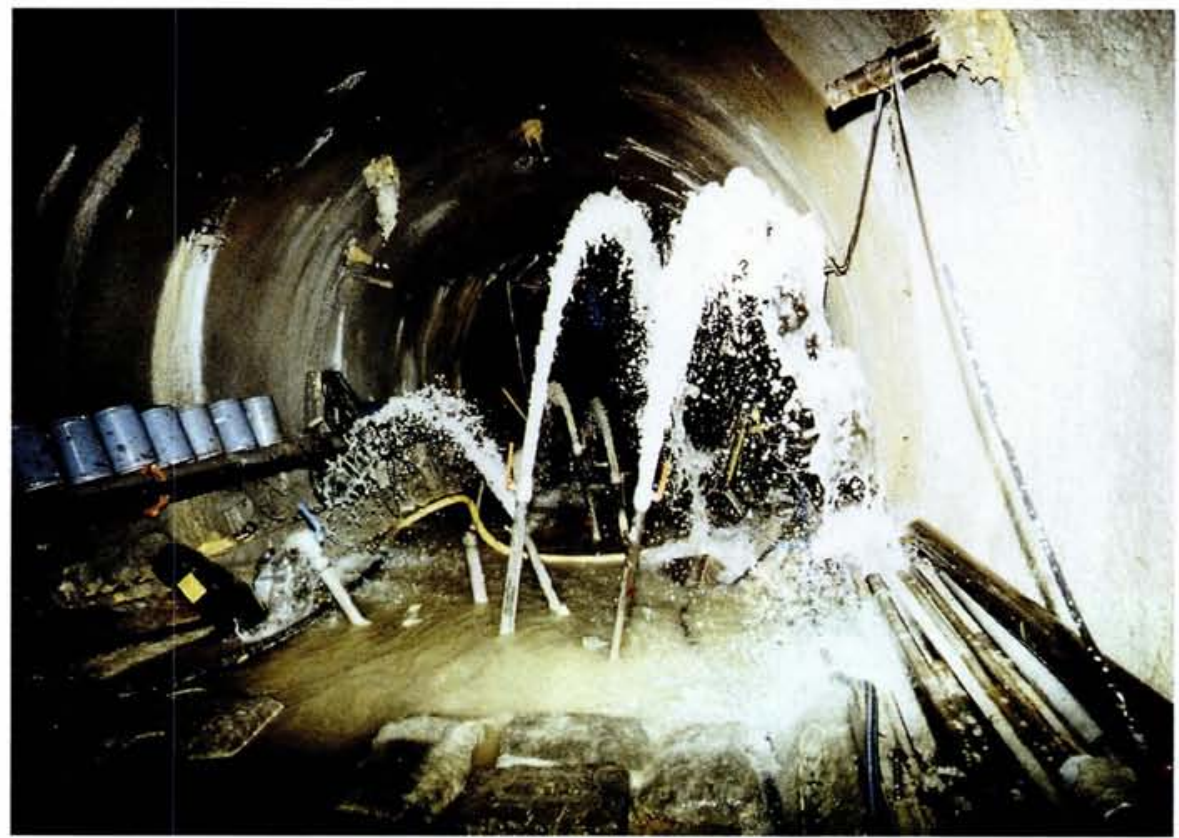

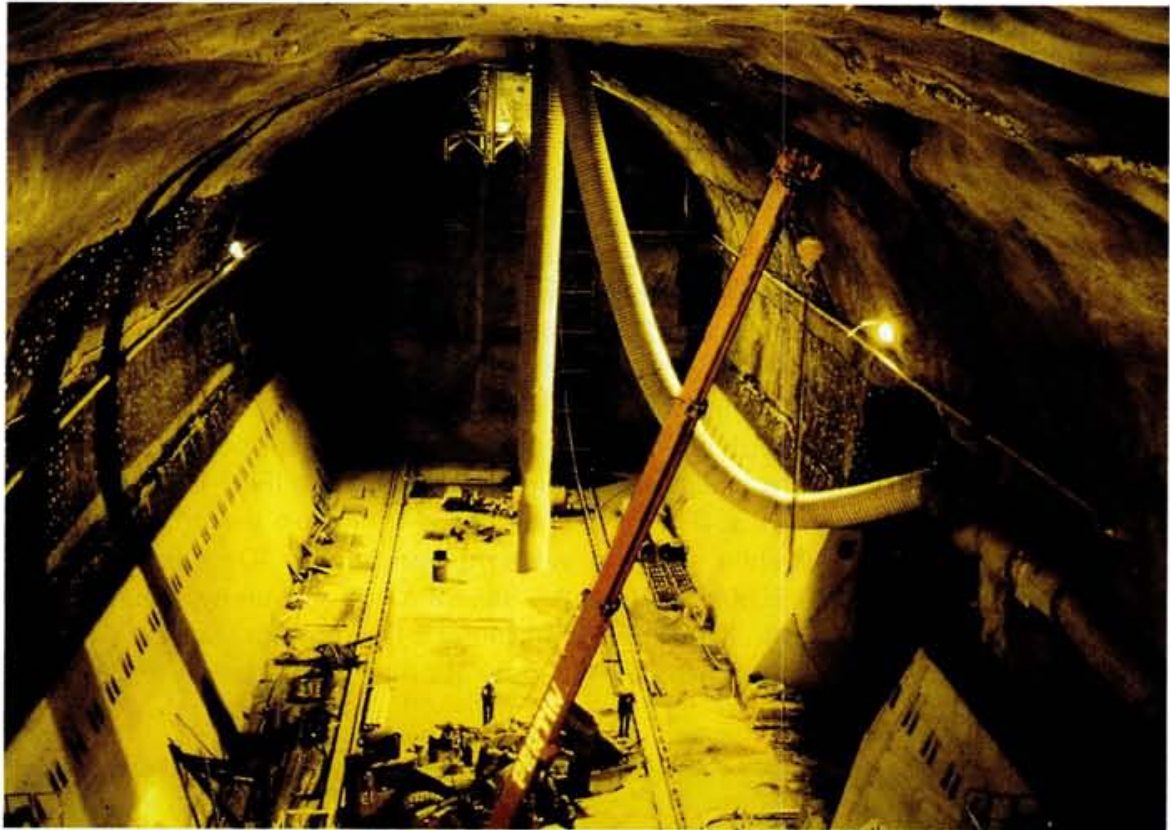

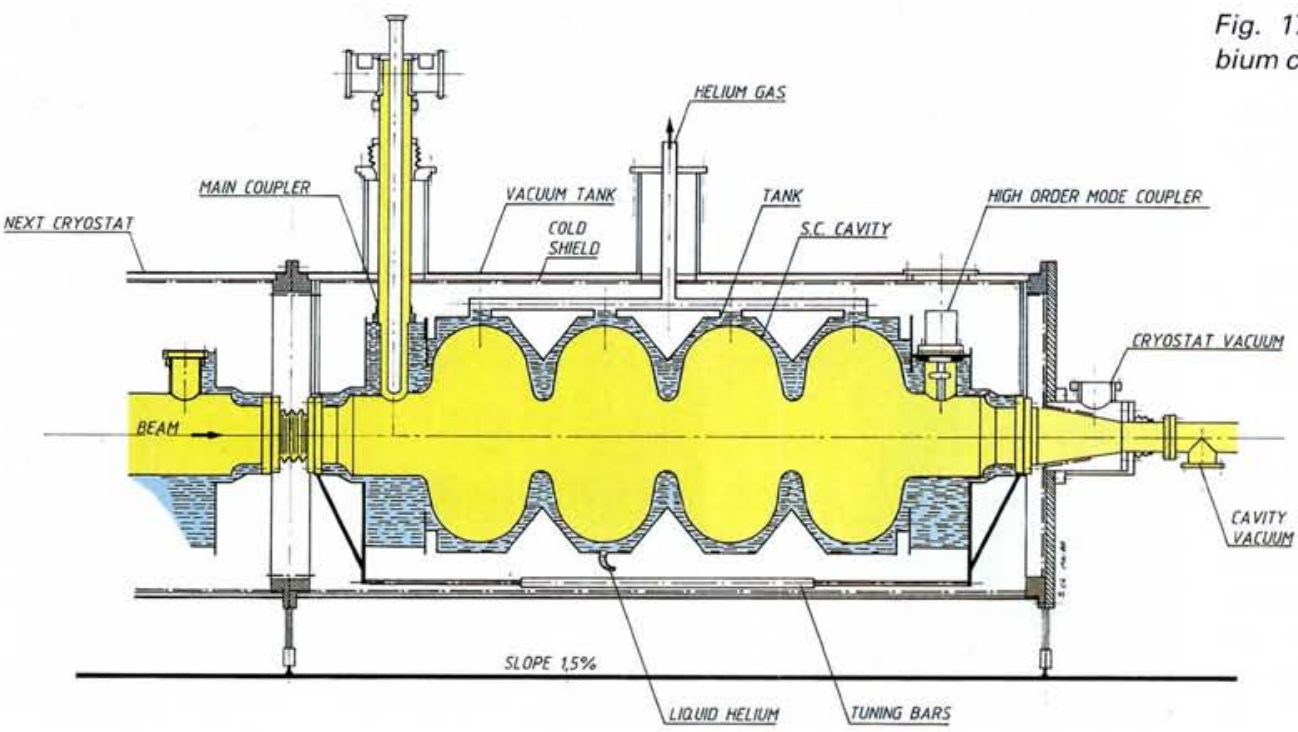

crossing points, the basic figure corresponding to the "half lattice period", i.e. the distance between the centres of two quadrupole lenses $(39.5 \mathrm{~m})$. The beam is most sensitive to alignment errors in the uniform fields of the quadrupole lenses, therefore these are used directly as survey monuments and all other elements are aligned with respect to them. The tolerance between nearby elements is of the order of $0.1 \mathrm{~mm}$. A quadrilateration chain between monuments more than $3 \mathrm{~km}$ apart has necessarily a certain "flexibility" and will, at the arc centre, deviate from the ideal orbit by the order of millimetres, a deviation that is acceptable for the beam provided local smoothness is maintained.

Fig. 15 (Left) - Civil engineering in an experimental cavern.

Fig. 17 (Below) - Superconducting niocavity.

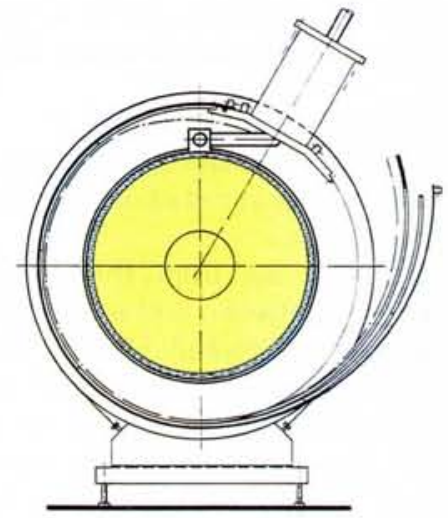




\section{Power and Cooling}

The power consumption of LEP will be about $80 \mathrm{MW}$ at $55 \mathrm{GeV}$ and $150 \mathrm{MW}$ at the top energy assuming that in the latter case, superconducting accelerating cavities will be used exclusively. Most of this power is dissipated in magnets and other electrical machinery or deposited by synchrotron radiation in the wall of the vacuum chamber, and must be carried away by a water cooling system of equivalent power capacity.

The power distribution system [4] which has been designed for the LEP complex, can all be derived from the 380 $\mathrm{kV}$ feeder line of the SPS. It is transformed down to $66 \mathrm{kV}$ and $18 \mathrm{kV}, 66 \mathrm{kV}$ cables feeding the major users, located in Points 2 and 6 for Phase 1, i.e. the power converters for the main dipole and quadrupole magnets, the acceleration system and the normal conducting solenoids of the experiments, and an $18 \mathrm{kV}$ loop around the whole machine tunnel providing power at $380 \mathrm{~V}$, via 24 substations, for all the "small" users.

The above-mentioned power consumers and the vacuum chambers (Fig. 4) are cooled [4] by demineralized water. The cooling loops in the arcs fan out from underground pumping stations in the even points, where the demineralized water is cooled by a primary loop which carries the heat up to the surface and into water/air heat exchangers (small cooling towers) of $10 \mathrm{MW}$ capacity each. The make-up water for the cooling towers comes from the waste water of the SPS which is channelled around LEP in pipes sunk into the floor of the machine tunnel.

In addition to the above-mentioned cooling circuits which work at temperatures between 20 and $60^{\circ} \mathrm{C}$, several $\mathrm{MW}$ of chilled water must be provided for the cooling of the electronic read-out systems of the experiments and the airconditioning of all the project.

\section{Ventilation and Air-Conditioning [4]}

The LEP tunnels and caverns must be ventilated so as to limit the concentration of ozone and $\mathrm{NO}_{x}$, both being produced by synchrotron radiation, and provide sanitary conditions for the workers. The air must be conditioned in particular in summer so as to avoid condensation and corrosion of the machine components. In the caverns, adequate ventilation capacity must be available for the case of major leakage of flammable or noxious gases out of the detectors. To establish the airflow necessary, the tunnels have to be divided into appropriate sections and control loops have to be set up over the $3,5 \mathrm{~km}$ distances between

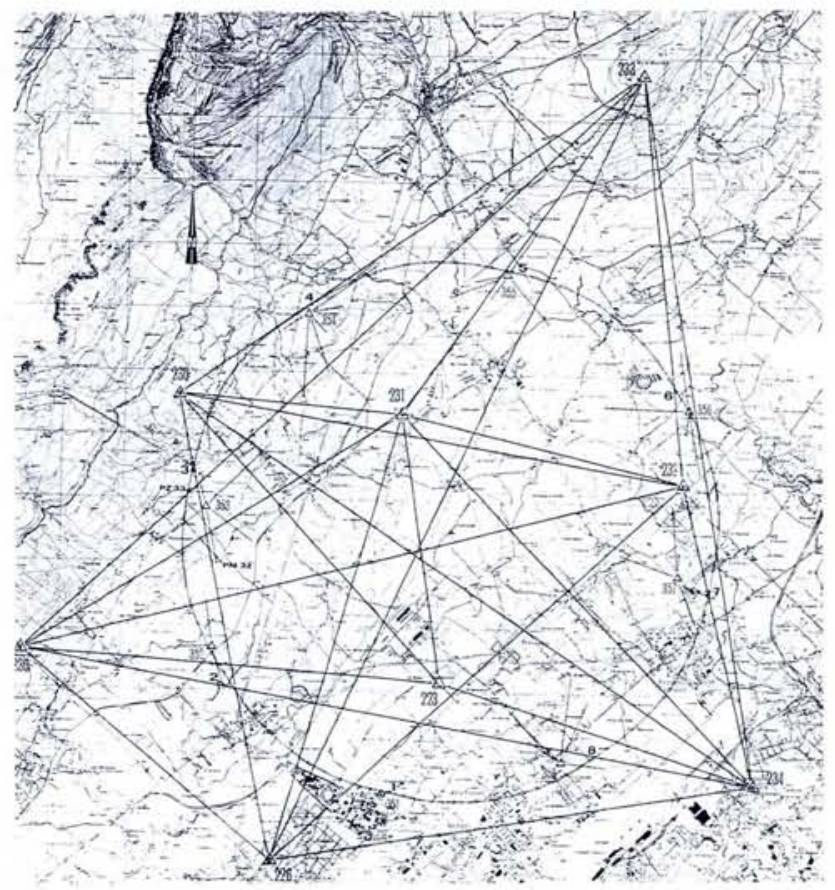

Fig. 16 - The Geodesic reference network.

air intake and outlet, so as to compensate the substantial differences in meteorological conditions which often occur. The total ventilation capacity installed is for 1.5 million $\mathrm{m}^{3} / \mathrm{h}$.

\section{Safety}

The risk of accidents is one of the major worries during the execution of a large project like LEP. A set of advisory committees on safety matters was active during the civil engineering and the installation phases, involving national safety authorities, representatives of the companies and of the workers, as well as outside advisors. The companies made great efforts in accident prevention, but still there occurred, regrettably, four fatal accidents underground during the civil engineering work. The installation activities were carried out, up to the date of writing, fortunately without accidents producing fatal consequences or even major injury.

For the finished machine, all normal precautions of safe engineering have been applied. There will be the usual failsafe radiation barriers at the tunnel entrances, access through them being controlled from the LEP control room. Flammable materials have been banned from the tunnel as far as this is possible. In particular, only cables with insulation respecting strict specifications in respect of fire propagation and radiation hardness have been admitted. All alarms involving danger to life are transmitted by hardwired circuits. However, in order to limit the amount of cable necessary, all subsidiary information is transmitted via the computers and the TDM links.

\section{ENGINEERING AND LOGISTICS}

For the LEP Project, a total of 60,000 tons of material had to be installed in the underground tunnels and caverns described above. Design, procurement and installation, the work of many dozens of firms and of CERN teams had to be coordinated, in particular with the civil construction activities. For these purposes, the use of informatics tools was introduced from the beginning.

\section{Informatics Support}

For the mechanical design work, a computer-aided design system was purchased and successfully used throughout [16], avoiding the hundreds of man-years of draughting effort required hitherto. The production of hundreds of installation plans, many of which are almost identical, was thus largely automatic, and the design of many complicated parts profited from the 3-D capabilities of the system.

A relational database system was used in conjunction with the CAD system as well as for many other applications, inventories for all parts and cables installed (permitting, on the basis of the stored information, the automatic routing of cables), subsidiary inventories and the overall PERT planning.

\section{Transport}

All material for underground installation has to be brought down one of the shafts and then transported along considerable lengths of the tunnel. Crane bridges with the necessary cable lengths are installed in the transfer halls on top of the pits, and a monorail system 
hanging from the ceiling so as to be less dependent on the floor occupation was provided in the machine tunnel. It was requested by the installation firms that all material be loaded in standard containers provided by CERN and delivered to the pithead, from where transport was organized by CERN according to schedules discussed in weekly meetings. Needless to say, the smooth operation of the transport service was one of the very essential backbones for timely termination of the project. The total distance covered by the monorail trains during LEP installation added up to $40000 \mathrm{~km}$.

The machine components in a strict sense, i.e. the magnets equipped with their vacuum chambers are the only exception to the above procedure. They were all assembled and aligned in one hall, picked up by the monorail, taken down through one specially large pit (the dipole units are almost $12 \mathrm{~m}$ long) and transported to their final location. The crane hook was fitted with a piece of monorail, so that there occurred no change of suspension while the alignment of the magnets was preserved.

\section{Project Planning}

The original planning differed a great deal from the execution sequence actually achieved. Originally, it was planned that tunnelling machines would start out essentially from the lowest point on the circumference and move upwards to join the Jura section, and infrastructure and machine installation would follow behind. However, a variety of problems led to a completely different sequence in the execution of the underground civil engineering and to a sizeable overall delay. Fortunately, very good preparation and a close control of the installation work permitted the machine installation to be adapted to the changing situations so that the project was finished within 7.5 years after Council approval, or more significantly only 6 years after ground breaking.

\section{DEVELOPMENT PLANS}

The basic layout of LEP is designed for a beam energy of $100 \mathrm{GeV}$, as discussed earlier in this report, the initial stage being chosen for a combination of scientific and financial reasons. Increasing the beam energy towards the design value is perhaps the most obvious line of development for LEP. Improving the luminosity, i.e. improving the statistical significance of the physics results or reducing the necessary running time, is definitely of the same importance. Finally both the optimism over achieving polarized beams in LEP and the interest in using these have increased substantially in recent years, and a programme is being defined in this direction [17].

\section{Energy Upgrade}

Based on early experience from the development of superconducting RF particle separators, a development programme aimed at the series production of superconducting accelerating cavities was launched at CERN about ten years ago, during the definition phase of the LEP project. Although a certain measure of understanding of the hitherto erratic behaviour of models at CERN and other laboratories had been gained by the time the LEP project was approved, it appeared unrealistic to rely at that time on a superconducting acceleration system. For the subsequent upgrade, to be implemented in the early nineties, the superconducting option was firmly foreseen [4].

As the thermal conductivity of the cavity is crucial for its quench behaviour, two lines of development have been followed: first, niobium sheet metal with much improved thermal conductivity has been obtained from industry, and second, the sputtering of niobium on the inside face of copper cavities has been developed. Prototype cavities (Fig. 17) satisfying the specified qualities, i.e. with an accelerating gradient $>5$ $\mathrm{MeV} / \mathrm{m}$ and a quality factor $>3 \times 10^{9}$ at this gradient, have been made at CERN using both techniques.

Today the influence of the various production steps on the quality of the cavity is pretty well understood, so that industrial companies are ready to submit firm offers for production series of superconducting cavities made from niobium sheet metal, and the first orders in the LEP upgrade programme [18] are being launched. There are three major milestones in this programme (Fig. 18). First, eight prototypes, four sheetmetal and four sputtered cavities, will be installed soon after the LEP start-up so as to gain experience with them in the machine environment. Second, enough cavities to run with the nominal beam current at the $Z^{\circ}$ production energy should be installed so as to save several million francs per year on the electricity bill, and third, the $W^{ \pm}$production threshold must be passed by a comfortable margin.

Because of synchrotron radiation, the circumferencial acceleration voltage installed $(U)$ must go up with the fourth power of the beam energy (E). When expressed respectively in $\mathrm{MV}$ and $\mathrm{GeV}$, this can be expressed as

$$
E=\text { const. } \sqrt[4]{ } U
$$

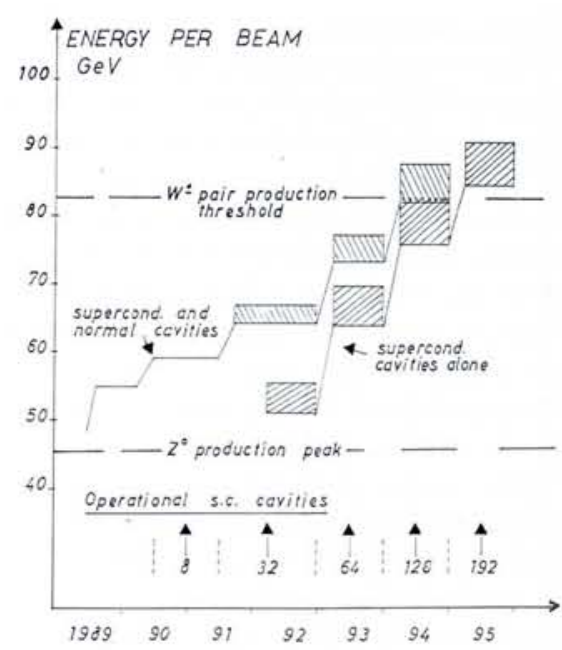

Fig. 18 - The energy up-grade programme for LEP.

where the constant ( $\cong 13$ ) depends on the focussing strength of the machine lattice. Table 2 gives the necessary numbers of cavities for a few typical cases within the upgrade programme.

Superconducting cavities present another important advantage over the warm copper cavities: because of the high ohmic losses, the copper cavities must be designed for a high shunt impedance at the expense of the coupling impedance seen by the beam, whereas the superconducting cavities can be designed with a large inner iris opening, leading to a smaller coupling impedance and a higher threshold current for beam instabilities.

While the production of the cavities is technically the most challenging part of the upgrade programme, many other systems have to be improved: klystrons must be set up to provide the RF power, certain types of magnets must be upgraded, many power supplies must be added in order to excite magnets to the required field levels, and klystron galleries must be dug at Points 4 and 8 . As shown in Fig. 18, this programme can be achieved by the end of 1993 if the necessary finance can be made available in the years to come.

\section{Luminosity}

The beam current, and hence the luminosity, in LEP is limited by the transverse mode coupling instability which sets a limit on the current contained in each bunch. The validity of the underlying assumptions and calculations will be tested as from the start-up of the machine and means are being studied to go beyond the assumed limitations. While it may be possible to improve the beam current by a factor of two by 
applying feedback or by changing the synchrotron oscillation frequency, a major increase of the total current can be achieved by an increase in the number of bunches circulating [19].

It is then necessary, because of the beam-beam tuneshift parameter, to avoid the additional beam crossings in the arcs. A scheme following work done at the CESR storage ring at Cornell University [20] where the beam orbit in the arcs would be deformed by additional electrostatic deflectors installed on either side of the collision areas has been computed for LEP [21]. It appears feasible to improve the luminosity by an order of magnitude at $Z^{\circ}$ energy once the acceleration power necessary for high energy operation has been installed, as this will then be available for the acceleration of the additional current at lower energy.

\section{CONCLUSIONS}

Having tried to give within the limits of this article a comprehensive review of the LEP Project, it has become very clear that it was impossible to go beyond basic sketches of the main lines of the project and to give just weight to the many man-years of thought, worries and dedicated hard work, and to the inventiveness of the staff, as well as the effort of the many industrial companies which participated in this enterprise. It was a real pleasure and an enriching and stimulating experience to collaborate with so many very competent and enthusiastic persons, to all of whom we are very greatful for their untiring dedication during all the years the project lasted. We hope now that all these efforts will be rewarded by a rapid commissioning and successful operation and a rich harvest of physics results.

The LEP collider together with the four experiments which are reported in the accompanying paper constitutes an instrument which will allow very precise experiments to be carried out. Although the "Standard Model" of modern particle physics, which unifies the description of the weak and the electro-magnetic interactions, has known a tremendous success, it still contains aspects not presented entirely satisfactorily. LEP will contribute to clearing these problems. History has also taught us that, though a priori justifications for scientific programmes have in general been valid for many years of research, in many cases new discoveries and unforeseen physical phenomena - new particles or new forces - have shown up, thus opening new horizons in the interpretation of the Laws of Nature.
Table 2 - Number of cavities for different collision energies

\begin{tabular}{|lc|c|c|c|c|c|c|c|c|}
\hline Number of SC Cavities & 8 & \multicolumn{2}{|c|}{32} & \multicolumn{2}{c|}{64} & \multicolumn{2}{c|}{128} & \multicolumn{2}{c|}{192} \\
\hline Acc. Gradient (MW/m) & 5 & 5 & 7 & 5 & 7 & 5 & 7 & 5 & 7 \\
Total Accelerating Voltage & 68 & 272 & 381 & 545 & 760 & 1090 & 1520 & 1635 & 2280 \\
Beam Energy (3 mA current) & & & & & & & & & \\
SC Cavities Alone & 36.3 & 51.3 & 55.8 & 64 & 69.5 & 76 & 82.6 & 84.1 & 91.4 \\
SC and Cu Cavities & 58.75 & 64.3 & 66.8 & 73.4 & 77.2 & 82.2 & 87.6 & 88.9 & 95.2 \\
\hline
\end{tabular}

\section{REFERENCES}

[1] See for example the Proceedings of the CERN Symposium, 1956.

[2] Amaldi U., Nuclear Instruments and Methods A243 (1986) 312-322.

[3] Courant W.D., Livingston M.S. and Snyder H.S., Phys. Rev. 88 (1952) 1190.

[3] The LEP Study Group, CERN/ISR-LEP/7817 (1978) CERN/ISR-LEP/79-33.

[4] The LEP Design Report Vol. 1, CERN-LEP. TH/83-29, Vol. 2, CERN-LEP/84-01.

[6] Lebrun Ph., Pichler S., Taylor T.M., Tortschanoff T. and Walckiers L., Design, Test and Performance of the Prototype Superconducting Quadrupole for the LEP LowBeta Insertions CERN-LEP-MA/87-51.

[7] Peschardt E. and Sladen J., CERN-LEPRF/89-29.

[8] 'The LEP Vacuum System', presented by H.P. Reinhard at the 9th International Vacuum Congress, Madrid (1983) LEP Note 449.

[9] Bovet C., Beam Diagnostics for LEP, CERN-LEP-BI/86-16.

[10] Isch H.W., Pett J., Proudlock P., 'An Overview of the LEP Power Converter System', Proc. IEEE Particle Accelerator Conference, Washington, D.C., March 1987, Vol. 3, p. 1399, CERN-LEP-PC/87-19.

[11] Innocenti P.G., Microprocessors in the LEP Control System, Proc. Int. Conference on the Impact of Digital Microelectronics and Microprocessors on Particle Physics, Trieste, March 1988, 73.

[12] Madsen J.H.B. et al., First Experience with the LEP Pre-Injector, Proc. IEEE Particle

Accelerator Conference, Washington, D.C., March 1987

[13] Laporte H., 'La Construction du LEP', Revue Travaux (Science et Industrie, Paris) 1988.

[14] Mayoud M., Proc. CAS School on Applied Geodesy for Particle Accelerators, 1986, CERN 87-01, 9 Feb. 1987.

[15] Genier C., Capper S., 'Logistics of LEP Installation', Proc. EPAC 1988 (to be published) and CERN-LEP-IM/88-19. Bachy G., Engineering and Construction Experience at LEP (CERN), Proc. Int. Ind. Symp. on the Super Collider (IISSC), New Orleans, February 1989, and CERN-LEP-IM/89-31.

[16] Colman K., Hauviller C. and Mottier M., La CAO pour la construction et l'installation du LEP, CERN-LEP-IM/84-05.

[17] Polarization at LEP Vols. 1 and 2, eds. G. Alexander, G. Altarelli, A. Blondel, G. Coignet, E. Keil, D.E. Plane and D. Treill, CERN 88-06.

[18] Bernard P., Lengeler H. and Picasso E., ddUpgrading of LEP Energies by Superconducting Cavities, presented by $\mathrm{H}$. Lengeler at the Workshop LEP 200, 29 Sept.-1 Oct. 1986, Aachen, and CERN-LEP-DI/86-29.

[19] Rubbia C., 'Future Physics at Accelerators', Proc. First European Particle Accelerator Conference, Rome, June 1988, to be published and CERN-EP/88-130.

[20] Littauer R., IEEE Trans. Nucl. Sci. NS-32 (1985) 1610.

[21] Jowett J.M., 'More Bunches in LEP', CERN-LEP-TH/89-17.

\section{The University of Stockholm Sweden}

\section{announces an opening for a \\ Full Professor of Nuclear Physics}

Salary depending on experience and personal qualifications. The letter of application supplied before 18 Sept. should be accompanied with a written account of scientific and teaching activities, a curriculum vitae, copies of degrees and other documents that the applicant wishes to supply, a numbered list of scientific publications and four separate parcels of these publications correspondingly numbered.

The documents should be addressed to:

The Registrar, University Administration

University of Stockholm

S-106 91 Stockholm

Sweden

For further information apply to the Registrar

(Tel. ++ (46) (8) 1620 00). 